\title{
Evaluation of the Binding Property of Some Binders in Metronidazole Tablet Formulation
}

\author{
Akodu Busayo Odunayo ${ }^{1}$, Fasuba Ilesanmi Kayode ${ }^{1}$, Akawa Ayodeji Benjamin ${ }^{2,}$, \\ Adu Isaac Adekola ${ }^{2}$, Olasehinde Oluwaseun Ruth ${ }^{2}$ \\ ${ }^{1}$ Department of Pharmaceutical Technology, University of Jos, Jos, Nigeria \\ ${ }^{2}$ Department of Medical Biochemistry, Afe Babalola University, Ado-Ekiti, Nigeria
}

Email address:

akawaab@abuad.edu.ng (A. A. Benjamin)

${ }^{*}$ Corresponding author

\section{To cite this article:}

Akodu Busayo Odunayo, Fasuba Ilesanmi Kayode, Akawa Ayodeji Benjamin, Adu Isaac Adekola, Olasehinde Oluwaseun Ruth. Evaluation of the Binding Property of Some Binders in Metronidazole Tablet Formulation. International Journal of Pharmacy and Chemistry.

Vol. 7, No. 2, 2021, pp. 22-30. doi: 10.11648/j.jpc.20210702.11

Received: February 17, 2021; Accepted: March 9, 2021; Published: March 30, 2021

\begin{abstract}
The binding property of five (5) commonly used binders in metronidazole granule and tablet formulations was studied. The binders studied included gelatin, polyvinylpyrrolidone (PVP), carboxymethylcellulose (CMC), maize starch and guar gum. The binders were incorporated as $5 \% \mathrm{w} / \mathrm{w}$ of tablet weight in each case. The granules were formulated by the wet granulation technique while the tablets were prepared by compression. The properties of granules evaluated included flow rate, angle of repose, bulk and tapped densities, Hausner's ratio, Carr's index and moisture content. Tablet properties studied included weight uniformity, hardness, friability, thickness and diameter, disintegration time and dissolution rate. Results indicated that granules containing guar gum or PVP possessed flow properties superior to those of other binders, while those containing gelatin possessed the least flow property. In terms of packing characteristics, granules containing CMC were better than others. The mechanical strength of tablets containing gelatin, PVP or CMC was superior to those made with either guar gum or maize starch. In terms of release of metronidazole, tablets containing PVP, gelatin or guar gum were more efficient in releasing the drug, while those made with $\mathrm{CMC}$ was the least. On the basis of these, good metronidazole tablets could be formulated using PVP, gelatin or guar gum. On the other hand, maize starch or CMC may not be recommended as a binder for metronidazole tablets because of high friability or poor release respectively.
\end{abstract}

Keywords: Binding Property, Metronidazole Granule, Polyvinylpyrrolidone, Carboxymethylcellulose, Tablet Formulations

\section{Introduction}

Binders are substances or agents used as tablet excipients to impart cohesive qualities to the powdered material during the production of tablets [1]. They ensure that the tablet remains intact after compression. They also improve the free-flowing quality of powders $[1,2]$. The choice of a particular binder depends on the binding force required to form granules and its compatibility with the other ingredients, particularly the active pharmaceutical ingredients $[1,2]$. Examples of binders used in tablet formulation are gelatin, starch (maize starch and potato starch), gums (acacia, guar gum), pre-gelatinized starch, cellulose derivatives (sodium carboxymethylcellulose, hydroxypropylmethylcellulose), cellulose, polyvinylpyrrolidone, polyethylene glycol, sucrose, methylcellulose, sorbitol and tragacanth [3]. There are four major sources of binders that are used in tablet formulation. They include binders from plant sources, animal sources semi-synthetic binders and synthetic binders [3]. Binders from plant sources could be obtained from any of sap, root, rhizome, leaves, fruit or seed from the parts of the plant [3]. These include starches, natural gums, and dried fruits while those from animal sources include gelatin, lecithin, chitin and lactose. Semi synthetic binders refer to those binders that are naturally-derived but have been chemically modified [4]. Examples are cellulose derivatives such as sodium carboxyl- methylcellulose, hydroxypropylcellulose, ethylcellulose, hydroxypropylmethylcellulose (HPMC) and 
methylcellulose $[4,5]$. On the other hand, synthetic binders refer to those binders that are derived from pure synthetic organic chemical substances $[4,5]$. Examples of synthetic binders are polyvinylpyrrolidone (PVP), polyethylene glycol (PEG), polyvinyl alcohol, poly methacrylate [5].

Metronidazole is widely used in tropical countries of Africa for the management of infections caused by susceptible anaerobic bacteria, some protozoan species and amoebiasis [6]. Metronidazole tablets in the market are found to exhibit one tablet defect or the other. These defects include hardness variation, capping, weight variation, cracking. These defects indirectly affect the efficacy of the tablet in delivering the drug. These problems are usually caused by inappropriate use of binders in tablet formulation $[5,6]$.

Tablets are intended for administration. Some are swallowed whole, some after being chewed, some are dissolved or dispersed in water before they are administered and some are retained in the mouth, where the active ingredient is released [7].

Today, among other dosage forms are widely prescribed today because they enabled an accurate dosage of medicament to be administered simply and they are potable. They are also more stable than liquid preparations and the release rate from tablets can be tailored to meet pharmacological requirements. Tablets are cost effective when compared with other dosage form $[7,8]$.

\section{Materials and Methods}

\subsection{Chemicals and Reagents}

Metronidazole BP, maize starch BP (Philip Harms Reagent, England), lactose (Roquett Pharmaceuticals, England), magnesium stearate (Gurr Chemicals GPR, Germany), gelatin (BDH Chemicals Ltd, England), hydrochloric acid (May and Baker Chemicals Ltd, England), polyvinylpyrrolidone, carboxymethylcellulose, guar gum, distilled water.

\subsection{Equipment}

The equipment used in this work included hardness tester (Monsanto, Erweka Germany), water bath (Techmel and Techmel, USA), disintegration tester (Eagle Scientific Ltd, England), Vanier caliper, Roche friabilator (Eagle Scientific Ltd, England), weighing balance, RC-6 Dissolution testing apparatus, single punch tabletting machine (Erweka EP-1, Germany).

\subsection{Preparation of Granules}

The wet granulation method was used in the preparation of granules according to the method of Rubinstein et al $[8,9]$. The quantities of materials required to produce fifty (50) tablets of metronidazole are shown in Table 1. To prepare the granules, a $10 \mathrm{~g}$ quantity of metronidazole powder (active ingredient) was weighed and transferred to a clean porcelain mortar. Then $11.5 \mathrm{~g}$ of lactose were weighed and added into the porcelain mortar. The two powders were triturated to obtain a homogenous mix. The binder solutions were prepared by dissolving or dispersing $1 \mathrm{~g}$ of each binder in sufficient volume of water to produce $5 \mathrm{ml}$ of solution or suspension. Each binder solution was added separately to the powder mix in the mortar. The mix was wet-massed to produce a damp coherent mass. In the case of gelatin, the solution was added while hot $\left(100^{\circ} \mathrm{C}\right)$. The maize starch was added as mucilage. The damp mass was wet screened by forcing them through sieve size 10 (corresponding to 2000 $\mu \mathrm{m})$. The resulting wet granules were dried in a drying cabinet at $60^{\circ} \mathrm{C}$ for $30 \mathrm{~min}$. The dried granules were passed through sieve size 16 (corresponding to $1190 \mu \mathrm{m}$ ). The granules were stored in an air-tight container $24 \mathrm{~h}$ before evaluation.

\subsection{Evaluation of Granules}

\subsubsection{Granule Flow Rate}

The flow rate of metronidazole granules was determined according to the method of Enauyatifard et al [10]. A $10 \mathrm{~g}$ quantity of each batch of granule was allowed freely through the orifice of a funnel mounted vertically by means of tripod stand and clamp. The time taken for each batch of granule to completely pass through the funnel was recorded. The flow rate was calculated using the formula:

$$
\text { Flow rate }=\frac{\text { Weight of powder }}{\text { Time of flow }}
$$

\subsubsection{Angle of Repose}

The heap of granules formed by each batch of granule from the flow rate experiment was used to determine the angle of repose. The height of the granule heap was measured using a ruler and broomstick. The contour of the heap was traced round with a pencil. The radius of the circle formed by each heap was measured by the ruler and was determined according to the formula:

Angle of repose $(F)=\tan ^{-1}(\mathrm{~h} / \mathrm{r})$ where $\mathrm{h}=$ height of the heap, $\mathrm{r}=$ radius of the base of the pile.

\subsubsection{Bulk and Tapped Densities}

A $10 \mathrm{~g}$ quantity of the granules was weighed and gently poured into a $100 \mathrm{ml}$ measuring cylinder. The volume attained after the granule was poured into the cylinder was taken as the bulk volume. The procedure was repeated three times and the mean bulk volume determined. The bulk density is the weight of powder divided by the bulk volume of powder. To determine the tapped density, the measuring cylinder carrying the granules was tapped gently but continuously on a smooth wooden platform until there was no further reduction in volume. This occurred after about 20 taps. The procedure was repeated three times and the mean tapped volume was determined. The tapped density was then calculated by dividing the weight of the powder by the mean tapped volume. 


\subsubsection{Carr's Compressibility}

This was derived by the application of the formula,

$$
\% \text { Carr'sCompressibility }=\frac{T d-B d}{T d} \times \frac{100}{1}
$$

\subsubsection{Hausner's Quotient}

This was derived by the use of the equation,

$$
\text { Hausner's Quotient }=\frac{\text { Tappeddensity }}{\text { Bulkdensity }}
$$

\subsubsection{Moisture Content}

A $2 \mathrm{~g}$ quantity of granules was weighed and poured into crucible placed in a hot air oven at $105^{\circ} \mathrm{C}$ for 30 minutes after which the content was reweighed. This was repeated at 30 minutes interval until there was no further loss of weight. The same procedure was followed for all the batches. The moisture content was calculated on dry weight basis using the equation:

$$
\% \text { Moisture content }=\frac{w_{1}-w_{2}}{w_{1}} \times 100 \%
$$

\subsection{Preparation of Tablets}

The die cavity of the tabletting machine (Erweka EP-1 Germany) was adjusted to hold granules corresponding to the target tablet weight of $500 \mathrm{mg}$ as stated in Table 1 above. The pressure settings of the machine were also adjusted to $6 \mathrm{MT}$. The granules of the various batches were first lubricated with magnesium stearate. They were compressed at the same weight and pressure settings. The machine was operated electrically to produce 20 tablets per min. A total of 50 tablets were produced from each batch of granules. The tablets were allowed to stand for 24 hours before evaluation.

\subsection{Evaluation of Tablets}

\subsubsection{Uniformity of Weight Test}

Twenty (20) tablets were randomly selected and individually weighed on an electronic balance (Mettler balance P 165, Greifensee-Zurich). The mean weight and the percentage coefficient of variation of each tablet were calculated. The percentage coefficient of variation was calculated for each batch using the formula the percentage coefficient of variation was calculated for each batch using the formula:

$\%$ coefficient of variation $=$ standard deviation $/$ mean $\times 100$

\subsubsection{Uniformity of Diameter and Thickness}

Five tablets randomly selected from selected from each batch were evaluated by measuring the diameter and thickness using a vernier caliper (Drapper Ltd, West Germany) individually. The mean diameter and thickness was then calculated and the standard deviation derived for each batch.

\subsubsection{Hardness Test}

Five (5) tablets were selected at random and the mean hardness determined using a Monsanto hardness tester (Eagle Scientific Limited, England). The mean and standard deviation values were calculated using the formula:

$$
T=\frac{2 P}{D t}
$$

Where, $\mathrm{P}=$ the load that causes tensile failure of a tablet, $\mathrm{D}=$ diameter of the tablet and $\mathrm{T}=$ tablet thickness.

\subsubsection{Friability Test}

Ten tablets were randomly picked from each batch and were collectively weighed. They were then placed in a Roche friabilator (Eagle Scientific Limited, England) and operated for 4 minutes at a speed of 25 revolutions per minute. The tablets were then dusted and reweighed and the percentage friability calculated according to the following equation:

$$
\% F=\frac{\text { Initial weight }- \text { final weight }}{\text { Initial weight }} \times 100 \%
$$

\subsubsection{Disintegration Time Test}

A $600 \mathrm{ml}$ volume of distill water was used as the disintegration medium. This medium contained in the disintegration apparatus (Eagle scientific Limited, England) was allowed to reach a temperature of $37^{\circ} \mathrm{C}$. The apparatus was calibrated to operate at 30 cycles per minute. Three tablets from a batch were dropped into each of the tablet cylinders and apparatus was started. The time taken for the last tablet or its fragment to pass through the mesh into the disintegration medium was recorded. The experiment was repeated for the other batches: the mean and standard deviation were then calculated.

\subsubsection{In-vitro Dissolution Test}

In-vitro dissolution test was carried out using the RC-6 dissolution testing apparatus. The dissolution medium was $1000 \mathrm{ml}$ of $0.1 \mathrm{~N} \mathrm{HCl}$ which was maintained at $37^{\circ} \mathrm{C}$. The machine was operated at 50 revolutions per minute. At time intervals of $30 \mathrm{~min}, 5 \mathrm{ml}$ samples of the dissolution medium were withdrawn. This was replaced with fresh $5 \mathrm{ml}$ of $0.1 \mathrm{~N}$ $\mathrm{HCl}$. The withdrawn samples were filtered before the absorbance reading was carried out using UV-Visible spectrophotometer (Romter, UV-1650PC, Shimadzu, Europe) The wavelength of maximum absorption used was $290 \mathrm{~nm}$. The per cent drug release at varying time intervals was determined from the slope of the calibration curve and plotted against time to generate the dissolution-time profile.

The concentration of drug released at each time interval is given by:

$$
\text { Concentration }(\mathrm{mg} \%)=\frac{\text { absorbance }}{\text { slope }}
$$

The per cent drug release at each time interval is given by: 
$\%$ drug release $=\frac{\text { concentration }(\mathrm{mg} \%) \text { at a given time }}{\text { final concentration }} \times 100 \%$

\subsubsection{Determination of Beer's Plot}

A standard calibration curve was determined by plotting the absorbance values generated from varying known standard concentrations against the standard metronidazole concentrations. A $100 \mathrm{mg}$ of the drug was accurately weighed using an analytical weighing balance (Mettler, Switzerland) and transferred to a $100 \mathrm{ml}$ volumetric flask. Then $20 \mathrm{ml}$ of $0.1 \mathrm{~N} \mathrm{HCl}$ was added to the flask to dissolve the drug. The volume was made up to $100 \mathrm{ml}$ with the dilute acid to produce a concentration of $100 \mathrm{mg}$ in $100 \mathrm{ml}$ of solution, representing a $100 \mathrm{mg} \% \mathrm{w} / \mathrm{v}$ solution.

This stock solution was then serially diluted by withdrawing $0.2,0.4,0.6,0.8$ and $1.0 \mathrm{ml}$ and making it up to $100 \mathrm{ml}$ with fresh $0.1 \mathrm{~N} \mathrm{HCl}$. This corresponds to concentrations of $0.2,0.4,0.6,0.8$ and $1.0 \mathrm{mg} \%$. The absorbances of these solutions were read on a UV-visible spectrophotometer (Romter, UV-1650PC, Shimadzu, Europe) at $290 \mathrm{~nm}$. A graph of absorbance versus concentration was plotted which gave the Beer's plot. The slope of the plot was determined. Since the plot is a straight line passing through the origin, it obeys the equation of a straight line given by:

$$
\text { Slope }=\frac{\text { Absorbance }}{\text { Concentration }(m g \%)}
$$

\section{Results}

The physical properties of batches of metronidazole granules produced with the various binders are shown in Table 2. The flow rate of the granules ranged from $1.78 \mathrm{~g} / \mathrm{s}$ for granules made with gelatin to $2.53 \mathrm{~g} / \mathrm{s}$ for those containing guar gum. Similarly, the angle of repose of the granules varied from $25.59^{\circ}$ for granules containing guar gum to $31.40^{\circ}$ for those made with gelatin. Both Hausner's ratio and Carr's index are derived from the bulk and tapped densities of the granules. Granules containing maize starch as binder possessed the least Hausner's ratio of 1.08, while those containing guar gum exhibited the highest Hausner's ratio of 1.14. On the other hand, granules containing maize starch had the least value of Carr's compressibility of $7.70 \%$, while those made with guar gum had the highest value of $12.50 \%$.

Angle of repose is used to estimate the flow of powders and granules. Generally granules possessing repose angles between $25-30^{\circ}$ usually exhibits good flow. Both Hausner's ratio and Carr's index are also used to estimate the flow of granules. Values of Hausner's ratio of 1.2 or below indicate good flow. Higher values represent cohesiveness of powders which result in poor flow. Carr's index is also used to predict the flow behavior of powders. Values of 5\% and below indicate good flow, while higher values represent poor flow of granules. On the basis of these, it can be seen that granules containing guar gum possessed the highest flow property, while those made with gelatin or maize starch possessed the least flow property. This result is in conformity with the flow rate of the granules.

The results for the physical properties of metronidazole tablets containing the various binders are shown in Table 3. The mean weight of batches of tablets ranged from $475 \mathrm{mg}$ for tablets containing maize starch as binder, to $512 \mathrm{mg}$ for those made with carboxymethylcellulose (CMC) as binder. The disintegration times of batches of tablets varied from $0.30 \mathrm{~min}$ for tablets containing polyvinylpyrrolidone (PVP), to $24.02 \mathrm{~min}$ for those made with CMC. It can be seen that all the tablet batches possessed the same diameter of 1.26 $\mathrm{mm}$. The mean thickness of batches of tablets ranged from $0.31 \mathrm{~mm}$ for tablets containing Gelatin as binder, to $0.34 \mathrm{~mm}$ for those made with maize starch as binder. Both hardness and friability values are used to assess the mechanical properties of tablets. They ensure that the tablet is able to withstand the stresses involved in packaging, transportation and handling of tablets without breaking. Tablets made with carboxymethylcellulose have the least friability of $0 \%$ while tablets made with maize starch have the highest friability of $3.73 \%$. On the other hand, the batch of tablet containing guar gum as binder has the least hardness of $5.8 \mathrm{kgf}$ and the batch of tablet containing gelatin has the highest hardness of 10.4 kgf.

In the dissolution profiles of metronidazole tablet, the calibration curve of metronidazole in $0.1 \mathrm{~N} \mathrm{HCl}$ at $37^{\circ} \mathrm{C}$ is shown in Figure 2. A straight line graph was obtained. The slope of the line graph was 5.7264 .

As shown in figure 2, the dissolution profiles of metronidazole from batches of tablets in $0.1 \mathrm{~N} \mathrm{HCl}$ at $37^{\circ} \mathrm{C}$, it can be seen that batches of metronidazole tablets containing gelatin, PVP or guar gum released almost all the drug contained in them within 2 hours. Batches containing maize starch or CMC did not attain $100 \%$ drug release within the time period.

As revealed in the dissolution parameters obtained from the dissolution profiles of batches of the tablets in table 4, the time taken for $50 \%$ of the drug to be released $\left(\mathrm{T}_{50}\right)$ ranged from $60.4 \mathrm{~min}$ for tablets containing PVP to $76.1 \mathrm{~min}$ for those made with CMC. The values of $\mathrm{T}_{90}$ (time taken for $90 \%$ of the drug to be released) varied from $108.7 \mathrm{~min}$ for tablets containing PVP to $137.0 \mathrm{~min}$ for those made with CMC. Similarly, the per cent drug released in $30 \mathrm{~min}\left(\mathrm{C}_{30} \%\right)$ varied from $19.7 \%$ for tablets containing CMC to $24.8 \%$ for those made with PVP. A similar trend of results was also obtained in the $\mathrm{C}_{45}$ and $\mathrm{C}_{60} \%$ of tablet batches, in which tablets containing $\mathrm{CMC}$ possessed the least values whereas those made with PVP exhibited the highest values.

$\mathrm{T}_{50}$ is the time taken for $50 \%$ of drug to be released, while $\mathrm{T}_{90}$ represents the time for $90 \%$ drug release. The lower the value of these parameters, the more efficient is the drug release from the tablets. On the other hand, $\mathrm{C}_{30}, \mathrm{C}_{45}$ and $\mathrm{C}_{60} \%$ 
represent the per cent drug released after 30, 45 and $60 \mathrm{~min}$ respectively. The higher the value of these parameters, the more efficient is the batch in releasing the drug from the tablet.

\section{Absorbance}

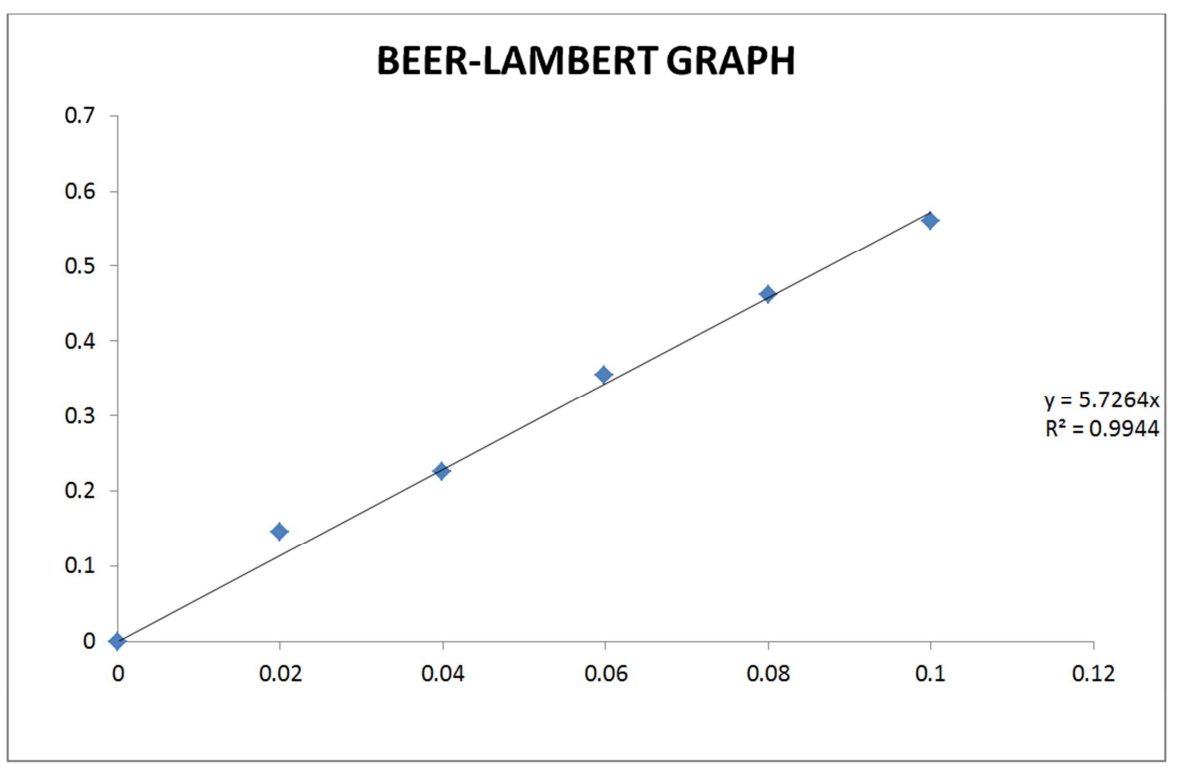

Concentration (mg \%)

Figure 1. Beer's plot of metronidazole in $0.1 \mathrm{~N}$ hydrochloric acid at $290 \mathrm{~nm}$ and $37 \pm 1^{\circ} \mathrm{C}$.

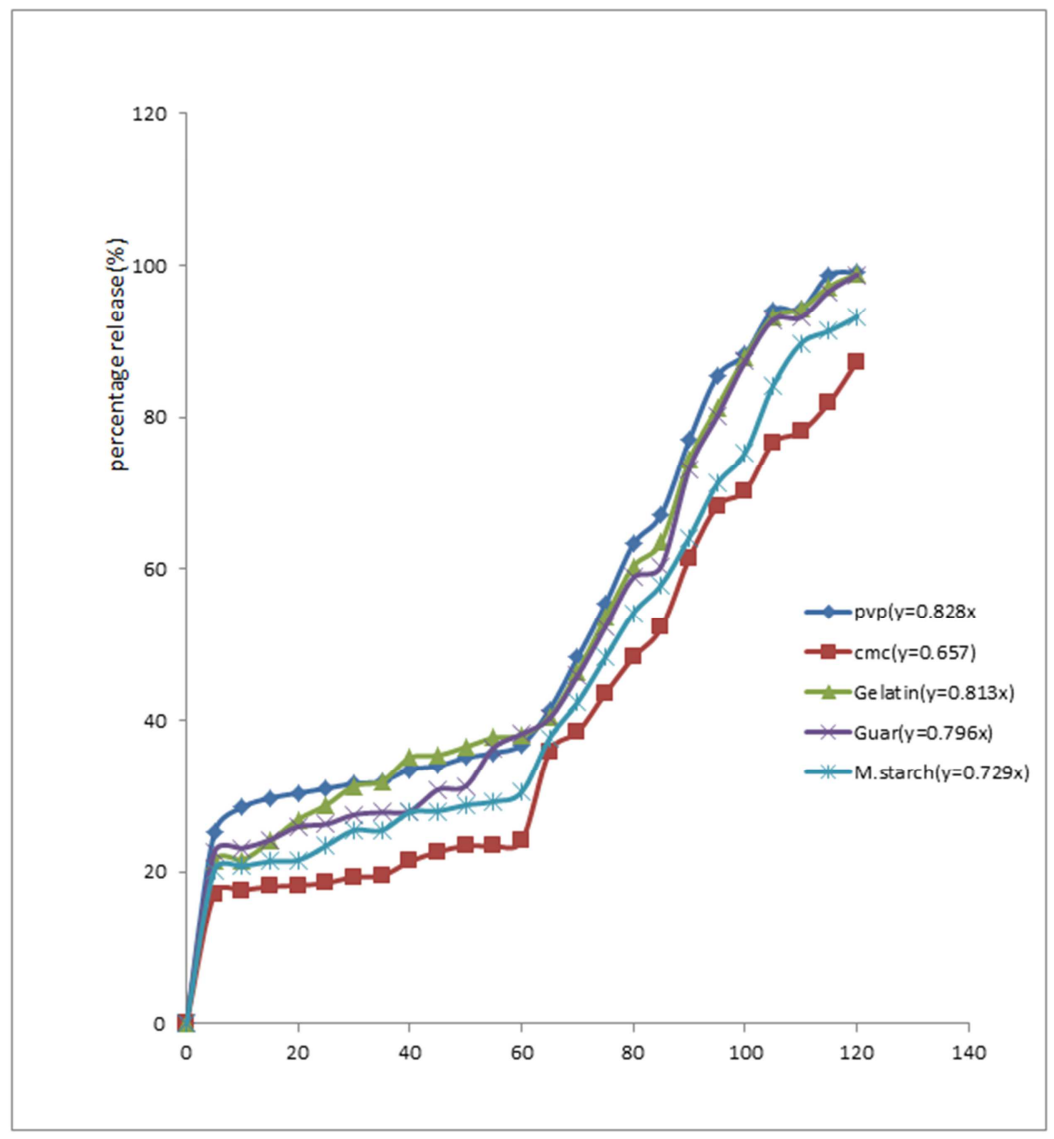

Time (mins.)

Figure 2. Dissolution profiles of batches of metronidazole tablets at $290 \mathrm{~nm}$ in $0.1 \mathrm{~N} \mathrm{HCl}$ and $37 \pm 1^{\circ} \mathrm{C}$. 


\section{Discussion}

In the physical properties and flow properties of batches of granules, the flow rate of granules containing guar gum was $2.53 \mathrm{~g} / \mathrm{s}$. This is the highest flow obtained among the various binders used. Granules containing gelatin possessed the least flow rate of $1.78 \mathrm{~g} / \mathrm{s}$. The flow rate of granules containing CMC, PVP, and maize starch are 2.28, 2.5 and $2.13 \mathrm{~g} / \mathrm{s}$ respectively. Flow rate determination gives an indication of the quantity of granules flowing through a channel within a given time. It is required that powders and granules employed in tablet formulation should flow well. Granulation is a method used to facilitate the flow of powders [11]. The shape, size and strength of granules formed by the binder used in granulation influence the flow of the granules. This in turn depends on the type and nature of binder used [11]. Moderately sized, spherical and strong granules tend to flow well, while fine, soft, irregularly shaped granules exhibit poor flow. When granules of similar size and shape are involved, the differences in the flow rate could be attributed to the strength of granules. This in turn is an inherent property of the binder used [12].

Angle of repose is a parameter used to predict the flow property of powders and granules. It has been generally established that angles of repose between $15-30^{\circ}$ indicate good flow $[12,13]$. Higher values result to poor flow due to cohesiveness of the powder [13]. The results of the angle of repose values obtained for the various batches are in conformity with those of the flow rate of granules. This is in conformity with the work of Elsabbagh et al [14].

Both Hausner's ratio and Carr's index are also used to predict the flow of powders and granules. It has been reported that as the value of Hausner's ratio approaches 1.2, the powder flows very well [14]. In the case of Carr's index, the lower the value, the better is the flow of granules. Whereas the results of the Hausner's ratios obtained for batches of granules follow similar trend as those of flow rate and angle of repose, those of Carr's index did not follow the trend as evident in the past work of Smith et al [15]. Overall, the flow property of granules increased in the order granules containing guar gum $>$ PVP $>$ CMC $>$ maize starch $>$ gelatin.

In the bulk densities of granules, it was revealed that the bulk densities of granules are $0.40,0.48$ and $0.42 \mathrm{~g} / \mathrm{ml}$ for granules containing gelatin, CMC and PVP respectively. The corresponding values for granules made with maize starch and guar gum are 0.38 and $0.42 \mathrm{~g} / \mathrm{ml}$ respectively. The values of tapped density of the granules are higher than the corresponding bulk density. Bulk density is an indication of the packing characteristics of powders and granules. Powders with high bulk density are advantageous in tabletting because of increased volume of fill in the die cavity of tabletting machines [16]. It was observed that the tapped density was greater than the bulked density. This is expected since part of the bed volume consists of voids which were removed by tapping. The bed diminished in volume since the void is eliminated $[15,16]$.

Table 1. Formula for preparing metronidazole 50 Tablet.

\begin{tabular}{|c|c|c|c|c|c|}
\hline \multirow{2}{*}{ INGREDIENT } & \multicolumn{5}{|c|}{ BATCHES (g) } \\
\hline & I & II & III & IV & $\mathbf{V}$ \\
\hline Metronidazole & 10 & 10 & 10 & 10 & 10 \\
\hline Lactose & 11.25 & 11.25 & 11.25 & 11.25 & 11.25 \\
\hline Guar gum & 1 & ----- & ----- & ----- & ----- \\
\hline PVP & ----- & 1 & ----- & ----- & ----- \\
\hline $\mathrm{CMC}$ & ----- & ----- & 1 & ----- & ----- \\
\hline Maize starch & ----- & ----- & ----- & 1 & ----- \\
\hline Maize starch (10\%) & 2.5 & 2.5 & 2.5 & 2.5 & 2.5 \\
\hline Magnesium stearate (1\%) & 0.25 & 0.25 & 0.25 & 0.25 & 0.25 \\
\hline
\end{tabular}

It can be seen that powders containing carboxymethylcellulose gave the highest values of bulk and tapped densities compare to the other batches. An increase in tapped density is an advantage in tabletting because of reduced volume of fill [17]. Both good flow and complete filling of die cavity would ensure uniformity of weight and content of compressed tablet [17]. The inter-particulate interactions that influence the bulk properties of a powder are also the interactions that interfere with powder flow. In freeflowing powders, these interactions are less significant and bulk and tapped densities will be closer in value. For poorly flowing materials, there are greater inter-particulate interactions and greater differences between bulk and tapped densities will be observed [18].

Table 2. Some physical properties of metronidazole granules containing the various binders.

\begin{tabular}{|c|c|c|c|c|c|}
\hline PROPERTY & GEL & CMC & PVP & MAIZE ST. & GUAR G. \\
\hline Flow rate $(\mathrm{g} / \mathrm{s})$ & 1.78 & 2.28 & 2.50 & 2.13 & 2.53 \\
\hline Angle of repose (degrees) & 31.40 & 26.60 & 26.60 & 29.31 & 25.59 \\
\hline Hausner's ratio & 1.09 & 1.11 & 1.10 & 1.08 & 1.14 \\
\hline Carr's compressibility (\%) & 8.00 & 9.50 & 8.34 & 7.70 & 12.50 \\
\hline Tapped density (g/ml) & 0.46 & 0.53 & 0.45 & 0.42 & 0.48 \\
\hline Bulk density (g/ml) & 0.40 & 0.48 & 0.42 & 0.38 & 0.42 \\
\hline
\end{tabular}

Key: $\mathrm{GEL}=$ Gelatin, $\mathrm{CMC}=$ Carboxymethylcellulose, $\mathrm{PVP}=$ Polyvinylpyrrolidone, MAIZE ST.=Maize starch, GUAR G. $=$ Guar gum 
The physical properties of batches of metronidazole tablets as shown in Table 2, reveals that the mean weights of tablets are 486, 512 and $509 \mathrm{mg}$ for tablets containing gelatin, CMC and PVP respectively. The corresponding mean weight values for tablets made with maize starch and guar gum are 475 and $510 \mathrm{mg}$ respectively. Weight uniformity is an important parameter of tablets as it ensures that tablets of uniform weight are produced in a batch. A coefficient of variance of not more than $5 \%$ is an indication of an excellent uniformity of weight for tablets weighing over $300 \mathrm{mg}$ (BP, 2010). These results showed that all batches of tablets complied with the compendia requirements for weight uniformity (BP, 2010). Weight uniformity minimizes the risk of overdosing or under-dosing of patients [18].

The physical properties of batches of metronidazole tablets containing the various binders were revealed in table 3 . Here, the hardness values of batches of metronidazole tablets are 10.4, 6.2 and $10.2 \mathrm{kgf}$ for tablets containing gelatin, CMC and PVP respectively. The corresponding hardness values for those made with maize starch and guar gum are 8.0 and 5.8 kgf respectively. On the other hand, the values of friability obtained for batches of tablets are $0.04,0$ and $0.08 \%$ for tablets containing gelatin, $\mathrm{CMC}$ and PVP respectively. The corresponding friability values for tablets containing maize starch and guar gum are 3.73 and $0.07 \%$ respectively. Both hardness and friability values are used to assess the mechanical properties of tablets. They ensure that the tablet is able to withstand the stresses involved in packaging, transportation and handling of tablets without breaking [19, 20]. Although hardness of tablets is not official in pharmacopoeias, values of $4-8 \mathrm{kgf}$ are generally accepted as adequate to ensure protection from mechanical stress and integrity of the tablets $[19,20]$. The friability of tablets is important, especially for low-dose and potent medicaments. Values of $0.1-1.0 \%$ are permissible [19].

Table 3. Some physical properties of batches of metronidazole tablets containing the variousBinders.

\begin{tabular}{llllll}
\hline Property & GEL & CMC & PVP & MAIZE S & GUAR G. \\
\hline Mean weight (mg) & 486 & 512 & 509 & 475 & 510 \\
Disintegration time (min) & 0.81 & 24.02 & 0.30 & 0.45 & 0.73 \\
Diameter (mm) & 1.26 & 1.26 & 1.26 & 1.26 & 1.26 \\
Thickness (mm) & 0.31 & 0.32 & 0.32 & 0.34 & 0.33 \\
Friability (\%) & 0.04 & 0 & 0.08 & 3.73 & 0.07 \\
Hardness (kgf) & 10.4 & 6.2 & 10.2 & 8.0 & 5.8 \\
\hline
\end{tabular}

Key: $\quad \mathrm{GEL}=$ Gelatin, $\quad \mathrm{CMC}=$ Carboxymethylcellulose,

$\mathrm{PVP}=$ Polyvinylpyrrolidone

MAIZE ST.=Maize starch, GUAR G.=Guar gum

Tablet thickness and diameter are measures of the size of tablets. The values of thickness and diameter of batches of metronidazole tablets are also shown in Table 3. All batches of tablets possessed the same diameter of $1.26 \mathrm{~mm}$ since the same punch size was used to produce them [21]. The thickness of the tablets, however, varied from $0.33 \mathrm{~mm}$ for tablets containing gelatin to $0.34 \mathrm{~mm}$ for those made with maize starch [21].
Tablet thickness is directly related to tablet hardness and can be used as an initial control of this parameter. Tablets which are too thin are liable to break easily while those which are too thick may be difficult to swallow. Manufacturers set limits on the thickness of tablets of various products in order to assure trouble-free packaging of tablets [20, 21].

It is recommended that tablet diameter and thickness should not exceed a coefficient of variance of 5\% depending on the weight [22]. The results showed that the thickness and diameter of all the batches fell within the accepted range. Hence all batches of tablets passed the tests for uniformity of thickness and diameter. This is in tandem with the work of Canton et al [21].

The disintegration times obtained for the batches of metronidazole tablets were also revealed in Table 3 and the values ranged from $0.81 \mathrm{~min}$ for tablets containing gelatin to 24.02 min for those made with CMC. Conventional uncoated tablets are expected to disintegrate within 15 minutes (BP, 1993) [22]. The results show that only tablets containing CMC failed the disintegration time test.

Disintegration involves the rapid breaking up of tablets into smaller fragments when the liquid penetrates the pores of the tablet [23]. It thus ensures that a tablet releases its active ingredient when exposed to the appropriate medium [23]. The smaller the granules size, the harder the tablet and subsequently the longer the disintegration time. The concentration of binder is a factor that affects the disintegration time profile [24]. If the binder concentration is high, it will increase the hardness of the tablets and consequently, the disintegration time will be increased [24]. The stronger the effect of binder, the more efficient must be the actions of the disintegrant or the tablet may not release its active ingredient at the proper time and place [24].

In figure 2, the dissolution profiles of batches of metronidazole tablets were taken into account. The dissolution parameters are shown in Table 4 . From the $\mathrm{T}_{50}$ and $\mathrm{T}_{90}$ values obtained, it can be seen that the batch containing PVP was the most efficient in releasing the drug from tablets. This is because this batch possessed the least values of both the $T_{50}$ and $T_{90}$ [24]. This is closely followed by the batches containing gelatin, guar gum, maize starch, while the batch containing CMC exhibited the highest values of both parameters.

Table 4. Dissolution parameters of batches of metronidazole tablets.

\begin{tabular}{llllll}
\hline Parameter & GEL & CMC & PVP & MAIZE ST. & GUAR G. \\
\hline $\mathrm{T}_{50}(\mathrm{~min})$ & 61.5 & 76.1 & 60.4 & 68.6 & 62.8 \\
$\mathrm{~T}_{90}(\mathrm{~min})$ & 110.7 & 137.0 & 108.7 & 123.5 & 113.1 \\
$\mathrm{C}_{30}(\%)$ & 24.4 & 19.7 & 24.8 & 21.9 & 23.9 \\
$\mathrm{C}_{45}(\%)$ & 36.6 & 29.6 & 37.3 & 32.8 & 35.8 \\
$\mathrm{C}_{60}(\%)$ & 48.8 & 39.4 & 49.7 & 43.7 & 47.8 \\
\hline
\end{tabular}

The dissolution of drug into the medium is necessary, in that only dissolved drugs can be absorbed to achieve its therapeutic effect [24]. The shorter time it takes for the drug to be released, the more drug will be available for absorption in the gastrointestinal tract [24]. On the basis of these dissolution parameters the binders can be ranked in order of 
increasing drug release as $\mathrm{PVP}>$ Gel $>$ Guar gum $>$ Maize starch $>$ CMC.

\section{Conclusion}

The binding property of five commonly used binders in metronidazole granule and tablet formulations was studied. The binders studied included gelatin, polyvinylpyrrolidone (PVP), carboxymethylcellulose (CMC), maize starch and guar gum. The binders were incorporated as $5 \% \mathrm{w} / \mathrm{w}$ of tablet weight in each case. The granules were formulated by the wet granulation technique while the tablets were prepared by compression. The properties of granules evaluated included flow rate, angle of repose, bulk and tapped densities, Hausner's ratio, Carr's index and moisture content. Tablet properties studied included weight uniformity, hardness, friability, thickness and diameter, disintegration time and dissolution rate.

Results indicated that granules containing guar gum or PVP possessed flow properties superior to those of other binders, while those containing gelatin possessed the least flow property. In terms of packing characteristics, granules containing CMC were better than others.

The mechanical strength of tablets containing gelatin, PVP or CMC was superior to those made with either guar gum or maize starch. In terms of release of metronidazole, tablets containing PVP, gelatin or guar gum were more efficient in releasing the drug, while those made with $\mathrm{CMC}$ was the least.

On the basis of these, good metronidazole tablets could be formulated using PVP, gelatin or guar gum. On the other hand, maize starch or CMC may not be recommended as a binder for metronidazole tablets because of high friability or poor release respectively.

\section{Recommendation}

Gelatin, PVP or guar gum may be recommended for use as binders in metronidazole tablet formulation. The use level of each binder should be $4 \% \mathrm{w} / \mathrm{w}$ of the target tablet weight.

\section{Conflicting Interest}

Authors declare no conflicting interest.

\section{Acknowledgements}

We are immensely grateful to all the scientist and technologist that participated in this research work for contributing their quota both in morals and in finance.

\section{References}

[1] Rehman, B. M., Wahed, M., Khondkar, P., Ahmed, M., Islam, R., Barman, R., Islam, M. A. (2018). Effect of Starch 1500 as a binder and disintegrant in Lamivudine tablets prepared by high shear wet granulation, Pakistan Journal of Pharmaceutical Sciences, 21 (23) 455-459.
[2] Mohammed, B. B., Isah, A. B., Apeji, Y. E. (2011). The role of acid-hydrolysed cassava starch as binders in paracetamol tablets, Journal of Pharmaceutical Research and Innovation, 4 (6): $9-12$.

[3] Shailendra, P., Agrawal, S., Lodhi, B. S. (2012). Natural binding agents in tablet formation, International Journal of Pharmaceutical and Biological Archives 3 (3): 466-473.

[4] Ronelle, R. (2004). Synthetic excipents challenges all natural organics offer advantages/ challenges to developers and formulators. Advenster Communications Inc 1: 38-50.

[5] Mukesh, G. (2016). Tablet binder's adhesives and granulating agent. Pharmainfo. Net, http://ww.pharmainfo.net/binders.

[6] Alebiowu, G. (2010). Assesment of tapioca starches obtained after different steeping period as binders in a paracetamol tablet formulation, Farmacia 58 (45): 341-52.

[7] Shivalingam, M. R., Kumaran, K., Jeslin, D., Reddy, Y. D., Tejaswini, M., Rao, C. M., Tejopavan, V. (2010). Cassiaroxburghii seed galactomannan: A potential binding agent in the tablet formulation, Journal of Biomedical Sciences Research 2 (23): 18-22.

[8] Rubinstein, M. H. (2005). Tablets in Phamaceutics, the science of Dosage form design, Churchill Livingstone, London 34 (45) 457-463.

[9] Alekha, K. D., Somnath, S., Tolman, J. (2014). Solid dosage forms in pharmaceutics basic principles and application to pharmacy practice, $1^{\text {st }}$ edition editors Elsevier Inc UK 987 (12): 161-162.

[10] Enauyatifard, R., Azadbakht, M., Fadakar, Y. (2012). Assessment of Ferulagummosagum as binding agent in tablet formulations, Acta Poloniac Pharma, Drug Research 6 (9) 291-8.

[11] Menon, S. S., Basavaraj, B. V., Bharath, S., Deveswaran, R., Madhaven, V. (2011). Formulation and evaluation of Ibuprofen tablets using Orange peel pectin as binding agent, Derm. PharmaciaLettre 3 (34) 241-7.

[12] Symeko, C. and Rhodes, C. T. (2014). Binder functionality in Tablet System Drug development and Industrial Pharmacy 21 (9) 1091-1114.

[13] Paul, J. J. and Eugene, L. P. (2016). Comparison of granules strenght and Tablet tensile strength. Journal of pharmaceutical sciences 72 (5): 530-531.

[14] Elsabbagh, H. M., Ghanem, A. H., Abdel-Alim, H. M. (2017). The influence of binders and concentration on the physical characteristics of calcium carbonate granules and their corresponding tablets. Pubmed Publications 36 (8): 548-51.

[15] Smith, J., Hong-Shum, K., Lily, L. (2011). Complex mixture of Phosphatidyl ethanolamine, Phosphatidyl inositol, Phosphatidyl acid, Glycolipids and Food additives data book $2^{\text {nd }}$ edition, Chichester, West Sussex Willey- Blackwell 334 (45) 23-32.

[16] Okafor, I. S. (2011). The Rheological Properties of Grewiagum, Nigeria Journal of Polymer Science and Technology 2 (65) 169-175.

[17] Ngwuluka, N. C., Idiakhoa, B. A., Nep, E. I., Ogaji, I., Okafor, I. S. (2010). Formulation and evaluation of Paracetamol tablets manufactured using the dried fruit of Phoenix dactylifera Linn as excipient Research in Pharmaceutical Biotechnology 2 (3) 25-32. 
[18] Agrawal, Y. K. and Prakasam, K. (2014). Effect of Binders on Sulfamethoxazole Tablets. Journal of Phamaceutical Sciences. 7 (10) $885-888$.

[19] Pifferi, G. and Restani, P. (2013). The safety of pharmaceutical excipients II Farmaco 58 (8): 541-550.

[20] Alderborn, G. (2013). Tablets and compaction in Aulton's Pharmaceutics. The design and manufacture of medicines, $4^{\text {th }}$ edition. Churchill Livingstone, London.

[21] Canton, S. L., Augsburger, L. L., Hoag, S. W., Armin, G.
(2018). Pharmaceutical granulation process, mechanism and the use of binders, research gate Publications 1 (54): 261-301.

[22] Dilip, M. P. (2014). Handbook of Pharmaceutical graduation technology CRC press, Florida $2^{\text {nd }}$ edition.

[23] Jones, D. (2018). Solid Dosage Forms1: Tablets in Pharmaceutics-The science of dosage form design, $1^{\text {st }}$ edition, Pharmaceutical press London. ISSN: 9780-85369-7640.

[24] Karthik, V. V. (2016). Excipients used in the formulation of tablets research and reviews. Journal of Chemistry. 5 (2): 143-154. 